\title{
Support for Australian carers from community pharmacy: insight into carer perspectives of a novel service
}

\begin{abstract}
The feasibility of an individualised carer support service delivered in community pharmacies was assessed from the perspective of carer participants using a pre-post questionnaire and semistructured interviews. Eligible pharmacies were required to offer a medication management service relevant to carers and have a semi-private space for conversations. Carers were required to self-identify as an unpaid support person for someone with a chronic condition or disability. Between September 2016 and March 2017, staff from 11 community pharmacies in South-East Queensland, Australia were trained, and provided with ongoing mentoring from a pharmacist and carer to support service implementation. Identification of carers and support to achieve a personal and caregiving goal were key features of the service. Questionnaires included the EQ5D-3L, the Bakas Caregiving Outcomes Scale, and questions relating to goal achievement, carer roles and responsibilities. Seven follow-up carer interviews were undertaken between March and May 2017 and analysed thematically. Pre-post questionnaires were available for 17 carers (1 withdrawn, 2 incomplete). Of the 29 goals set, 10 were achieved and 14 partially achieved. EQ-5D-3L scores were unchanged, while seven of the 15 items comprising the Bakas score improved $(\mathrm{p}<0.05)$. Carer service evaluation was generally favourable, and these two main interview themes were the impact of caring and pharmacy experience. The impact of caring, whilst variable, was significant. Pharmacy experiences were mostly positive and the opportunity for carers to further engage with pharmacy staff was appreciated. The service was feasible and initial reported benefits to carers may support further research potentially in terms of a larger controlled trial.
\end{abstract}

Keywords: caregiving, pharmacy, pharmaceutical care, feasibility 


\section{What is known about this topic}

- Informal carers are an integral part of healthcare and social support in the community, contributing to significant cost-savings for Governments.

- Community pharmacy staff assist carers with managing medication for the person they provide care for and other medication related issues.

- The negative impact of the caring role on wellbeing is well researched; while support interventions focus on managing the carer's role rather than their own health needs.

\section{What this paper adds}

- A service provided by community pharmacy staff is feasible and may assist carers to achieve personal and caregiving goals and other positive outcomes, in particular those related to their caregiving role.

- Overall, carers were satisfied with the support they received from pharmacy staff.

- This study indicated that progression to a larger, controlled trial is warranted. 


\section{Introduction}

Informal carers including family, are integral to providing healthcare in the community, particularly with an ageing population and limited long-term formal care services (World Health Organisation, 2016). The global prevalence and circumstances of these carers is unknown; however, it is evident that this population is substantial, with an estimated 43.5 million adult carers in the United States [US] (AARP Public Policy Institute \& National Alliance for Caregiving, 2015), 6.8 million carers in the United Kingdom [UK] (Buckner L \& Yeandle S, 2015) and 2.86 million self-reported carers in Australia (Deloitte Access Economics \& Carers Australia, 2015). Yet, even with a high carer population, relevant research is inconsistent and fragmented (Solomi \& Casiday, 2016).

A significant role for informal carers is medication management for the person/s they support (Noureldin \& Plake, 2017). A systematic review by Parand et al. (2016) explored the types of medication errors associated with carers' administration; dosing inaccuracies were most common. Carers may face other medication-related issues, such as ensuring medication adherence, particularly when caring for people with late-stage dementia (Alsaeed et al., 2016). Canadian researchers identified that risk of medication interactions was of particular concern for carers (Williams et al., 2016). Studies in the US (Noureldin et al., 2017, Look \& Stone, 2017) and UK (Maidment et al., 2017, Smith et al., 2003) have emphasised the important role of community pharmacy staff assisting carers, especially concerning medication management. Pharmacists, as medication experts, have opportunities to assess carers' involvement in medication-related tasks and address associated problems (Noureldin \& Plake, 2017). Yet, limited research has occurred within the context of community pharmacies, which is surprising given they are accessible, local, healthcare destinations, and pharmacists have a wide scope of practice beyond medication supply. 
To our knowledge, the only pharmacy service that has focused on supporting carers was piloted in the UK; staff were trained to identify carers and refer them to appropriate healthcare or carer services (Mooney G \& Wigfield A, 2015). In Australia, there is considerable support available from Government services, including financial assistance and access to Aged Care/Home Support assessments, as well as from non-government organisations, such as Carers Australia. However, as seen internationally, carers and pharmacists may not be aware of, or have access to, such services (Willemse et al., 2016). Ploeg et al. (2017) also identified that some carers were unaware of the support available, or that they may resist access to such services for a number of reasons, such as a personal belief that they were coping reasonably well or reluctance from the care-receiver to have others involved. A recent audit confirmed these findings for a sample of Queensland (Australian) carers, in addition to cultural and financial barriers (Walbank S, 2017). Health professionals need to be aware of these factors, and be proactive towards recognising carers in the community rather than relying on carers to self-identify their role and responsibilities (Knowles et al., 2016).

Overall, there is a substantial amount of research on the impact that caring for someone can have on carers' wellbeing (Adelman et al., 2014). This is commonly referred to as 'carer burden', and while this is not to say that caring lacks positive aspects (Carbonneau $\mathrm{H}$ et al., 2010), the concept of burden is a 'central theoretical construct within almost all carer research' (Lawn \& McMahon, 2014). Yet, support interventions frequently do not address the carer's health needs, focusing instead on assisting them to cope with their caring role (Corry et al., 2015). The paucity of research calls for an individualised support intervention that is focused on the carer's needs by exploring not only their caregiving role, but takes into account their personal health and wellbeing. As pharmacists can provide a range of professional services to assist carers, such as arranging Home Medicine Reviews (Carter et al., 2016), and 
interact regularly with carers who manage medication, pharmacists could be ideal for providing such a service.

In this study, the UK carer pilot (Mooney G \& Wigfield A, 2015) was adapted to meet the Australian context, and extended with the incorporation of goal setting and follow-up sessions with trained pharmacy staff to assist carers to meet their goal/s, which included medication concerns or queries. Although not specifically a medication management service, goals could be related to medication concerns or queries. Further details about how the service was developed and implemented within the pharmacy setting are published elsewhere (McMillan SS et al., 2018). The aim of this study was to assess the feasibility of an individualised carer support service in the community pharmacy setting, from the perspective of carer participants.

\section{Methods}

Study Setting

University Ethics approval was obtained (GU Ref No: 2016/014). Pharmacists and pharmacy assistants $(\mathrm{n}=39)$ from 11 community pharmacies in South-East Queensland were trained in September-October 2016 to deliver the carer support intervention. This location was selected to allow researchers and two mentors (a carer and a pharmacist) easy access to visit participating pharmacies for support purposes. Pharmacy recruitment involved a variety of methods, such as contacting professional colleagues, conference networking and email advertisements from professional pharmacy organisations. Eligible pharmacies were required to have a semi-private space to speak with carers in the pharmacy, such as a consultation room or a quiet area where conversations were not overheard by other customers, as well as offer medication-related services of relevance to carers. For example, if a staff member identified 
that a carer could use assistance with organising their medication, then a dose administration aid could be initiated.

The carer support service

A minimum of two interested pharmacy staff members (pharmacists, interns or assistants) from each pharmacy was provided with face-to-face training; this included insights into the caring role, information on local support services, and research processes including consent. For example, staff were asked to reflect on what they understand by the term carer and the factors that may have influenced a person to step into this role, as well as to record their thoughts and/or reactions to videos which highlighted the daily lived experience of carers. Discussions of how staff could support carers accessing their pharmacy occurred, including sharing of previous experiences of working with carers and associated challenges. Mentors also provided follow-up support as needed and the inclusion of a young carer in this process was critical in that it ensured that ongoing feedback and support was delivered in a carer context that extended beyond other pharmacy services, e.g. medication review and counselling. Promotional material, such as posters and postcards were given alongside carer folders to record questionnaire responses and follow-up notes. Carer folders outlined the six steps of the service: (i) research consent process for the carer (not the care-receiver); (ii) carer health status (i.e. getting to know the carer such as their roles and responsibilities); (iii) initial health review, including medication/s used and impact of caring; (iv) support plan (goal setting and strategies informed by answers to the carer health status and initial health review); (v) ongoing support (such as follow up sessions); and (vi) final review (McMillan SS et al., 2018). A mentor pair visited or phoned each pharmacy twice-monthly for follow up purposes; support focused on carer recruitment and goal setting, and access to resources. 
The service was provided at no cost to carers; pharmacies were remunerated for their time (\$50 AUD for each completed carer folder). Using the carer folder, a trained pharmacy staff member worked with each carer to explore their caring role and personal wellbeing (steps two and three) using the EQ-5D-3L General Health Questionnaire (EUROQOL, 2013) and the Bakas Caregiving Outcomes Scale (Bakas et al., 2006). Carers were asked to indicate the type of support they provided to the care-receiver, e.g. role-related tasks such as emotional support, medication management, etc. The EQ-5D-3L was chosen to assess carers' quality-of-life as it is a widely used and validated generic measurement. After considering the range of caregiver assessment measures available (Family Caregiver Alliance \& Benjamin Rose Institute on Aging, 2012), the 15-item Bakas scale was deemed most suitable to evaluate the impact of caregiving (although it has only been validated for specific carers, e.g. of stroke survivors). Carers were asked about the number and frequency of medications they personally used for their own wellbeing, as well as medications used by the person they supported (i.e. carereceiver). After initial (pre) assessment, staff worked with carers to set two goals - one related to their caring role and the other related to their personal wellbeing, and negotiated strategies to achieve these goals. Follow-ups (face-to-face, phone or email) could occur at any time as individually required, for example, when the carer came into the pharmacy to collect medication over the next three months. An end-of-service (post) evaluation was undertaken as part of the final review (Step 6 in the carer folder) to assess goal achievement, perceptions of the service, impact on caregiving outcomes, etc. Participants were asked to repeat The EQ-5D3L General Health Questionnaire (EUROQOL, 2013) and the Bakas Caregiving Outcomes Scale (Bakas et al., 2006). Further details of service feasibility with pharmacy staff are reported elsewhere (McMillan SS et al., 2018).

Study participants 
Trained pharmacy staff were asked to recruit and work with six adult carers per pharmacy, for up to three months depending on the carer's individual needs. Carer participant numbers allowed the researchers to explore the feasibility of the service within funding constraints. Pharmacy staff were advised to recruit people they knew to be carers, as well as consider others who may be in a caring role, e.g. people picking up medication or dose administration aids for others. To participate, carers were required to self-identify as an unpaid support person for an individual with any chronic condition or disability; people who were formally employed as paid caregivers were excluded. Written consent was obtained from carers to participate in the service. Verbal consent was acquired to audio-record all interviews and these participants received a \$20 AUD gift voucher to thank them for their time.

\section{Data collection}

The service was implemented between September 2016 and March 2017. Completed carer folders were collected for analysis purposes, following which carers were interviewed by telephone about their experience. Exploratory interview questions (Table 1) were developed with reference to the literature (Wilson et al., 2013, Willemse et al., 2016, McCann \& Bamberg, 2016) and feedback from the research team (which included a researcher with lived experience as an adult carer) and key stakeholders, such as an advisor from Carers Queensland and a young carer; this diversity broadened the range of carer perspectives. Interviews were undertaken between March and May 2017 by an experienced qualitative researcher (SM); one carer preferred to provide written feedback to questions due to personal time constraints. A transcribing company transcribed interviews, which were de-identified and quality checked by the interviewer.

Data analysis 
Carer folder responses were entered into SPSS v21 (IBM, New York, USA) and data reported using descriptive statistics. Changes were tested in the EQ-5D-3L, EQ VAS and 15-item Bakas scale data between the two-time points (pre/post service). The EQ-5D-3L scores were converted to EQ-5D weights for Australia (Viney et al., 2011) and the weighted scores compared using a Wilcoxon Signed Ranks Test. The total Bakas score was identified according to scoring instructions (approval obtained), with missing data imputed using the mean of the available scores (one value was missing from three participants post-service). Pre and post scores were compared for the general health state (EQ VAS) and total Bakas score were compared using a paired sample t-test. Individual items in the Bakas Scale were compared pre and post using a Wilcoxon Signed Ranks Test. A $p$-value of $<0.05$ declared a statistically significant difference.

The interview transcripts were read by the interviewer and coded in relation to the interview topics. Some key themes emerged from the data when comparing transcripts, i.e. thematic analysis. A second researcher (AS) read the transcripts to confirm the analysis. As participant numbers were limited to carers that participated in the service, interviewing until data saturation was not possible. Hence, the qualitative information presented in this manuscript is classified more broadly within two key themes: the impact of caring and pharmacy experiences. Quotes were labelled to represent the carer participant from a specific pharmacy, i.e. P6_04 was the participant that provided written feedback, and represents the fourth carer from the sixth recruited pharmacy.

\section{Results}

Demographic results 
Twenty carer folders were returned to researchers; results from one carer who withdrew were not used. All 19 carers self-reported being White Australian; the majority were female $(n=14)$ and cared for one family member $(n=16)$. The mean carer age was 61.1 (range 27-78 years). The average caring role for the seven carers interviewed was 10.8 years (range 2.5-25). Eight participants worked part-time and six were retired. Eighteen carers self-reported providing emotional assistance, with seven of these carers also assisting with household duties, medication management, healthcare, and physical and financial assistance. The most common health professional involved in the carer's support team was the General Practitioner (GP), followed closely by the pharmacist; nine carers visited a community pharmacy on a weekly basis. Fifteen carers self-reported personally taking one or more medications (range 1-7; mean $=4.36$; median $=4.50$ ), with care-receivers taking a median of seven medications (range 2-15).

Initial health review and carer follow-up

Seventeen of 19 carers completed both pre/post questionnaires. No reason was provided in the carer folders as to why two participants did not complete the service. However, one of these carers rated their overall health state as 95 ( $0=$ worst health state; $100=$ best health state) and hence may not have perceived a need for the service; the other carer supported three people and may have had limited time to fully participate. Sixteen of the 17 carers who completed the service recorded one or two goals, with a total of 29 goals set by participants. Goals focused on the carer personally encompassed areas such as weight loss and making time for themselves, including physical exercise, shopping and family time. Carer-related goals were based on improved symptom control for the care-receiver and obtaining information on respite or home help. The number of pharmacy follow-ups ranged between zero and four per carer (median 3; total 43). The majority of follow-ups were undertaken face-to-face $(n=28 ; 77.8 \%)$, phone $(n=7$; 19.4\%) and email ( $n=1 ; 2.8 \%)$. 
Carer outcomes and service evaluation

A third of goals were achieved $(n=10)$ and half partially-achieved $(n=14)$. There was no change in general quality of life (EQ-5D-3L weighted scores; $p=0.285$ ) or general health (EQ VAS; $\mathrm{p}=0.739$ ) pre/post service delivery (see Supplementary Information Table 1). A significant improvement in participants' total Bakas score was obtained after service completion ( $p=0.013)$. Of the 15-items in the Bakas scale (see Supplementary Information Table 2), seven showed statistically significant improvement post-service: i) financial wellbeing ( $p=0.036)$; ii) self-esteem ( $p=0.033$ ); iii) time for family activities ( $p=0.007)$; iv) relationship with friends ( $p=0.014)$; $v$ future outlook ( $p=0.027)$; vi) roles in life ( $p=0.001)$; vii) time for social activities with friends $(p=0.041)$. Overall, positive results were obtained from the service evaluation (Table 2).

Interview findings: the impact of caring and pharmacy experiences

The personal impact of caring was varied with discussion mostly directed towards the burden rather than the positive aspects of caring. This encompassed changes to future plans, health, lifestyle and self-identity:

...that's the unfortunate thing because you lose your own identity as well. You kind of forget who you are and what, what you used to do (P6_05)

One participant explained that while she still loved her husband, their marriage had changed:

...I've lost my husband. He's sort of like a companion now...I was happily married but I'm really not in a marriage now. It's not... a loving normal marriage...I mean I love my husband dearly and I've decided that my role is to care for him now while he's still here...(P2_07) 
Alternatively, another participant described her spousal role as encompassing caring responsibilities:

...I see it [being a carer] more as just a natural extension of what a wife would do... (P6_03)

Three carers described particular challenges or frustrations they faced, for example, when ablebodied individuals used toilets or parking spaces for people with disability. Another carer described snapping back at her cognitively impaired husband and then feeling guilty. The following participant discussed bureaucratic difficulties she experienced as a carer for her adult daughter:

...you can't have a say because she [daughter] is an adult, yes, but she's an adult with not capacity... (P2_02)

Some participants revealed their discomfort in talking about themselves, or mentioned coping strategies to manage care-recipient behaviour:

I generally sort of take the focus off me pretty quick and (laughs)...I've just sort of fallen into that thing that it's never about me...(P6_05)

One carer discussed feelings of isolation as her social network consisted solely of her husband whom she cared for. Another participant described having a strong support network from people in a similar position to her:

I'm a member of the Vietnam Veterans' Ladies Group and once a fortnight, we have lunch...That's [a] really good time, because we all have the same problems and we can all help one another (P5_06) 
Carers described mostly positive pharmacy experiences. Two carers were grateful for assistance when they had run out of prescription medication, either for the care-receiver or themselves:

...I'd run out of warfarin tablets and I didn't have another script, and we couldn't get to the doctor until the following week and I went down to the chemist...and straight away she said I'll give you your warfarin tablets, don't worry about it. Ah, when you get your script from your doctor just drop it in ... (P8_04)

This was the only carer who described pharmacies providing further support with continuing medication supply; they questioned whether pharmacies would ever be given the authority to issue prescriptions for life-long medication. Other forms of assistance were discussed, such as home deliveries, dose administration aids, and organising new prescriptions, which helped alleviate the burden associated with treatment.

The important role of pharmacy staff in ensuring safe medication supply was emphasised by one carer, who had experienced a medication error. This participant, who was also a midwife, explained that while she acted as a safeguard by picking up the error, her concern was for other consumers less knowledgeable about medication. While medication information and reassurance about safety and appropriateness were viewed as core pharmacy services, a potential role to provide information about other services and access to specialised equipment or resources was highlighted. For example, one carer experienced difficulty accessing catheter bags, and recommended that pharmacies signpost carers to other information and supports:

...maybe that even could be something that the pharmacy could let carers know that there are these services [e.g. Transcord], rather than an ad hoc way of getting something in your letterbox. (P6_03) 
Another carer initially questioned whether it was the place of pharmacy staff to provide anything other than medication-related information. However, further into the interview when they were asked about whether the carer service fitted within the pharmacy setting, there was some agreement that the pharmacy could provide limited guidance about other services:

...if they can see that someone who was needing extra support then having the understanding and the knowledge of where to guide them, I think that's because the pharmacy is the first port of call for a lot of people...even just having basic contacts for different areas to be able to help guide anybody in that direction if they need to. (P2_02)

This ambiguity reflects the opinions of some carers who, while not sure that the service fitted within the pharmacy setting, were unsure where the service was best situated. One carer responded with the following question: if you didn't put it in the pharmacy, where else? (P8_04). Questions were raised as to whether pharmacy staff had sufficient resources and time to provide the service. Adequate space was needed to facilitate confidential conversations, and the suitability of the pharmacy was discussed in terms of pharmacy brand or size:

...Smaller pharmacies can do all of that [provide care] because they, you build up that personal relationship and they know whether you're going down or up or you know getting a little bit depressed or whatever. They can see those things, whereas a large pharmacy, you're just another number going in and out (P6_02)

Another carer, who was previously a pharmacy auditor, believed that carers needed to develop rapport with a pharmacist:

...if you build up a rapport with them, they've got a good understanding of the medication that the...person you're caring for is on. They can say "oh [name] you know 
the doctor's prescribed such and such but look, you know when I look at this, that's going to interact with this other medication”... (P2_07)

Building this relationship was seen as a way to facilitate conversations around a carer's wellbeing and increase awareness of pharmacy services. Another carer emphasised that it took a special type of person to be able to approach carers and initiate a conversation. Ultimately, being empathetic and actively listening were key attributes for pharmacy staff:

...often people just want to talk...all they need, is somebody to give them some time and if somebody can have some empathy and listen, they need to be good listeners... (P5_06)

Telling a carer that they needed to put their health first was not necessarily the best approach: ...they [GP] say you know, 'it's very important that you look after yourself'...if I hear that one more time I'm going to scream... (P2_07)

While no suggestions were made to change the service, it was evident from the interviews that the impact was limited in this population but that there were benefits realised. These included the pharmacy staff knowing more about a carer's situation, acknowledgment that the support was available (beyond medication), and for some, a recognition that they were either managing their carer responsibilities or needed to make some changes:

It [the service] made me realise the emotional impact, that's (laugh) yeah, which is probably an unusual answer but...until you actually really sort of start speaking about it and people sort of start asking certain questions relating to it, you sort of think oh wow, I didn't realise how, you know, how this affects me emotionally. (P6_05) ...it [service] showed me that I am not, resting enough and I'm doing too much...so I've backed off on some of my church responsibilities (P5_06) 
My first thought was...how much time will it involve, as I have nil spare...I didn't know at that time that I too would benefit greatly...Help was always at hand. The staff are always most obliging and helpful. Advice was always given to assist. I received the encouragement I needed to focus on my own health and understand the importance of doing that (P6_04)

\section{Discussion}

Research has recognised the impact of caring on the carer's own physical and mental health (Happell et al., 2017), and constraints in attending to their own healthcare needs (Shrank et al., 2011). Our study did not shed any new insights on the impact of caring. However, it was revealed that a carer support service within a community pharmacy setting can facilitate a number of positive outcomes, though these were limited to the overall caring role rather than the carers' personal wellbeing. For some participants, the service provided an opportunity to reflect on their situation and identify if any changes were needed.

There was some ambivalence about the role that pharmacy staff could perform beyond carer recognition and medication supply. Some carers could visualise pharmacies assisting with some aspects of care coordination, such as access to services, while others questioned whether this was within the scope of pharmacy practice. While there are many factors influencing the implementation of Australian community pharmacy services (Hossain et al., 2017), consumer expectations and perceptions of the pharmacist's role can hinder or enable service success. Further work in showcasing to carers what pharmacists can do for them, beyond medication supply, is warranted. It is also evident that community pharmacy services need to meet the needs of the individual consumer, as well as the existing gaps in healthcare practice (Hossain et al., 2017). With respect to our study, there was some consensus that this individualised 
service was suitably placed within the pharmacy setting, with an acknowledgement that some carers may need more support than others, although they may be resistant to obtaining support for various reasons, such as pride or independence (Ploeg et al., 2017). An important component of equipping pharmacy staff to deliver a carer support service would be to include strategies that support staff to explore where carers are situated in their individual journey, and to be able to respond accordingly. As an accessible point of healthcare, community pharmacy is well placed to explore carer resistance; however, as evident in other healthcare settings (Burridge et al., 2017) organisational barriers need to be addressed, such as time and space.

The majority of carers in our study self-reported taking medication for their own health. This finding is consistent with that of an Australian survey about pharmacy services; 139 of the 160 recruited carers also identified as being a health consumer (Whitty et al., 2015). This result emphasises the need for healthcare professionals to view carers beyond their caring role; this must extend beyond simplistic questions such as 'are you looking after yourself,' as carers already acknowledge and recognise this as problematic (Solomi \& Casiday, 2016). In our study, one carer was clearly frustrated when advised by a doctor to take care of themselves. The need for more awareness of, and concern for, the health of carers by healthcare professionals such as GPs was recognised by a recent Australian study (Happell et al., 2017). However, this is a complex topic, with tension seen between a carers' willingness to prioritise their own wellbeing above others (Burridge et al., 2017).

While our service was designed to support carers' wellbeing by setting individualised goals, there was no change in carer's personal wellbeing (quality of life or general health scores) postservice. This could be attributed to the small sample size; the information gained will support sample size calculations for a future study. While we decided to report all carer-related findings 
in this paper, the lack of difference is somewhat expected given the study design. Although there were more obvious changes in Bakas scores somewhat related to caregiving roles, whether this can be linked to the service cannot be determined. Many positive experiences could also occur as part of the normal service provided within the pharmacy, which could not be separated from the study results. However, what this study does provide is some important preliminary insights into how carers perceived the service, therefore supporting a controlled effectiveness study. Currently, it is unknown if the intervention is associated with no improvement in the general quality of life of carers, etc., or if this is due to study design limitations.

Concerns or issues about managing medication, as evidenced by the extensive literature available on this topic (Look \& Stone, 2017, Maidment et al., 2017, Wingham et al., 2015, Alhaddad et al., 2016), was noticeably absent from our data. This was surprising given that pharmacists are well placed to address medication-related issues. Only one carer questioned whether pharmacists could formally provide medication without a prescription, i.e. medication continuance. It is possible that carers in our study had no medication-related concerns, or had any previous concerns addressed before participating in the study, or did not provide this level of assistance to the care-receiver; four out of the 19 carers did not report providing a medication-related role in the carer folders. Also, pharmacists may have provided medication assistance within the realm of their standard practice so carers did not expect any difference. Alternatively, the service as currently designed may not have placed enough emphasis on this specific topic, especially if staff were focused on carer goals. Other intervention studies to prevent carer-related medication errors will be used to inform future adaptations of this service. For example, Parand et al (2016) made numerous suggestions, such as checking carer understanding of medication labelling, storage and administration techniques. To our 
knowledge there is no formal training for informal carers with respect to medication management; including this aspect into a pharmacy delivered carer service may be particularly relevant to those carers managing multiple medications for the care-receiver.

\section{Limitations}

The results of this study should be viewed within its limitations; a small sample of Caucasian carers was recruited and the number of carers approached within each pharmacy, (i.e. the overall response rate), is unknown. The authors acknowledge the difficulties with carer recruitment and offer some insights into this, which are published elsewhere (McMillan SS et al., 2018). Many carers were conveniently selected by pharmacy staff to participate, leading to a possible sampling bias. No data were obtained as to why two carers did not complete, and why one withdrew from, the service. This has some implications on the feasibility of the service, although there are many reasons why carers may not have perceived the service to be relevant to them at that particular point of time. We were unable to reach data saturation given the limited number of participants available to be interviewed; while key themes were identified and are noteworthy in their own right, further research is needed to explore this topic in more depth. Information on uptake rates and testing of outcome measures for relevance and sensitivity is also required. The possibility of non-consent and non-response bias should be considered in future carer research (Oldenkamp et al., 2016); results need to be confirmed in a larger study given self-selection bias for pharmacies and carer participants. There was limited data in the carer folders on goals, strategies and follow-up discussions; alternative methods to obtain this data should be considered, such as an online platform. There is the risk of selfreported bias, particularly when responding to survey questions in front of, or alongside, a pharmacy staff member. However, the information obtained by the follow-up interviews, 
which were undertaken by an experienced qualitative researcher using a standardised interview guide, strengthened survey results.

While this is the first study that we know of that explored the feasibility of a personalised, goaldriven service for carers within the community pharmacy setting, there are a number of learnings that the researchers have made with respect to the intervention design. For example, no information was obtained in the carer folders as to how long a participant had been a carer, and there was no direction to pharmacy staff to include the care-receiver in the service (care/care-receiver dyad). Carers may have chosen to involve the care-receiver, but it is not known if, or to what extent, this occurred. While we did not ask carers to specify their own, or their care-receiver's individual health concerns, a strength of this study was that it was inclusive of carers for people with any chronic condition/s.

\section{Conclusion}

Carers who visit community pharmacies may benefit from an individualised support service that focuses on their needs as well as their caring role. This study showed some promising results that may be worthy of further exploration in a larger trial. Carers acknowledged what the service was trying to do, and while not suited for everyone, the opportunity to further engage with a pharmacy staff member was appreciated. Further work is needed to refine the service design, particularly in relation to medication management, which was not reported as an outcome of this pharmacy driven service. 


\section{References}

AARP Public Policy Institute \& National Alliance for Caregiving (2015) Caregiving in the U.S. Available at: https://www.aarp.org/content/dam/aarp/ppi/2015/caregiving-intheunited-states-2015-report-revised.pdf (accessed on 14 December 2017).

Adelman, R. D., Tmanova, L. L., Delgado, D., Dion, S. \& Lachs, M. S. (2014) Caregiver burden: a clinical review. Journal of the American Medical Association 311 (10), 10521060.

Alhaddad, B., Taylor, K. M., Robertson, T., Watman, G. \& Smith, F. J. (2016) Assistance of family carers for patients with COPD using nebulisers at home: a qualitative study. European Journal of Hospital Pharmacy 23 (3), 156-160.

Alsaeed, D., Jamieson, E., Gul, M. O. \& Smith, F. J. (2016) Challenges to optimal medicines use in people living with dementia and their caregivers: A literature review. International Journal of Pharmaceutics 512 (2), 396-404.

Bakas, T., Champion, V., Perkins, S. M., Farran, C. J. \& Williams, L. S. (2006) Psychometric testing of the revised 15-item Bakas Caregiving Outcomes Scale. Nursing Research 55 (5), 346-355.

Buckner L \& Yeandle S (2015) Valuing Carers 2015: The rising value of carers' support. Carers UK, The University of Scheffield, University of Leeds, Centre for International Research on Care, Labour \& Equalities. Available at: https://www.carersuk.org/forprofessionals/policy/policy-library/valuing-carers-2015 (accessed on 14 December 2017).

Burridge, L., Mitchell, G., Jiwa, M. \& Girgis, A. (2017) Helping lay carers of people with advanced cancer and their GPs to talk: an exploration of Australian users' views of a simple carer health checklist. Health \& Social Care in the Community 25 (2), 357-365.

Carbonneau H, Caron C \& Desrosiers J (2010) Development of a conceptual framework of positive aspects of caregiving in dementia. Dementia 9 (3), 327-353.

Carter, S. R., Moles, R., White, L. \& Chen, T. F. (2016) The willingness of informal caregivers to assist their care-recipient to use Home Medicines Review. Health Expectations. 19 (3):527-542.

Corry, M., While, A., Neenan, K. \& Smith, V. (2015) A systematic review of systematic reviews on interventions for caregivers of people with chronic conditions. Journal of Advanced Nursing 71 (4), 718-734. 
Deloitte Access Economics \& Carers Australia (2015) The economic value of informal care in Australia in 2015. Available at:

http://www.carersaustralia.com.au/storage/Access\%20Economics\%20Report.pdf (accessed on 14 December 2017).

EUROQOL (2013) The EQ-5D-5L and EQ-5D-3L. Available at: www.euroqol.org (accessed on 13 July)

Family Caregiver Alliance \& Benjamin Rose Institute on Aging (2012) Selected Caregiver Assessment Measures: A Resource Inventory for Practitioners. Available at: https://www.caregiver.org/selected-caregiver-assessment-measures-resourceinventory-practitioners-2012 (accessed on 14 December 2017).

Happell, B., Wilson, K., Platania-Phung, C. \& Stanton, R. (2017) Physical health and mental illness: listening to the voice of carers. Journal of Mental Health 26 (2), 127-133.

Hossain, L. N., Fernandez-Llimos, F., Luckett, T., et al. (2017) Qualitative meta-synthesis of barriers and facilitators that influence the implementation of community pharmacy services: perspectives of patients, nurses and general medical practitioners. BMJ Open 7 (9).

Knowles, S., Combs, R., Kirk, S., Griffiths, M., Patel, N. \& Sanders, C. (2016) Hidden caring, hidden carers? Exploring the experience of carers for people with long-term conditions. Health \& social care in the community 24 (2), 203-213.

Lawn, S. \& McMahon, J. (2014) The importance of relationship in understanding the experiences of spouse mental health carers. Qualitative health research 24 (2), 254266.

Look, K. A. \& Stone, J. A. (2017) Medication management activities performed by informal caregivers of older adults. Research in Social and Administrative Pharmacy. doi 10.1016/j.sapharm.2017.05.005 [Epub ahead of print].

Maidment, I. D., Aston, L., Moutela, T., Fox, C. G. \& Hilton, A. (2017) A qualitative study exploring medication management in people with dementia living in the community and the potential role of the community pharmacist. Health Expectations. doi 10.1111/hex.12534 [Epub ahead of print].

McCann, T. V. \& Bamberg, J. (2016) Carers of older adults' satisfaction with public mental health service clinicians: a qualitative study. Journal of Clinical Nursing 25 (11-12), 1634-1643. 
McMillan SS, King MA, Stapelton H, Sav A, Wheeler AJ \& F., K. (2018) A pharmacy carer support service: obtaining new insight into carers in the community. International Journal of Pharmacy Practice doi 10.1111/ijpp.12454 [Epub ahead of print]

Mooney G \& Wigfield A (2015) Carer-Friendly Pharmacy Pilot: Summary of the Evaluation Results. Carers Trust. Available at: https://professionals.carers.org/carer-friendlypharmacy-pilot-evaluation (accessed on 13 July 2017)

Noureldin, M., Murawski, M. M., Mason, H. L., Hyner, G. C. \& Plake, K. S. (2017) The association between family caregivers' involvement in managing older adults' medications and caregivers' information-seeking behavior. Journal of the American Pharmacists Association 57 (2):170-177.

Noureldin, M. \& Plake, K. S. (2017) Correlates of caregivers' involvement in the management of older adults' medications. Reearch in Social and Administrative Pharmacy 13 (4), 840-848.

Oldenkamp, M., Wittek, R. P., Hagedoorn, M., Stolk, R. P. \& Smidt, N. (2016) Survey nonresponse among informal caregivers: effects on the presence and magnitude of associations with caregiver burden and satisfaction. BMC Public Health 16480.

Parand A, Garfield S, Vincent C \& BD, F. (2016) Carers' Medication Administration Errors in the Domiciliary Setting: A Systematic Review. PLoS ONE 11 (12), e0167204.

Ploeg, J., Matthew-Maich, N., Fraser, K., et al. (2017) Managing multiple chronic conditions in the community: a Canadian qualitative study of the experiences of older adults, family caregivers and healthcare providers. BMC Geriatrics 17 (1), 40.

Shrank, W. H., Liberman, J. N., Fischer, M. A., et al. (2011) Are caregivers adherent to their own medications? Journal of the American Pharmacists Association 51 (4), 492-498.

Smith, F., Francis, S. A., Gray, N., Denham, M. \& Graffy, J. (2003) A multi-centre survey among informal carers who manage medication for older care recipients: problems experienced and development of services. Health \& Social Care in the Community 11 (2), 138-145.

Solomi, V. L. \& Casiday, R. E. (2016) In sickness and in health: The strains and gains of caring for a chronically ill or disabled spouse. Chronic Illness 13 (2), 75-87.

Viney, R., Norman, R., King, M. T., et al. (2011) Time trade-off derived EQ-5D weights for Australia. Value Health 14 (6), 928-936.

Walbank S (2017) Quality of Life Audit 2017 Report. Carers Queensland Ltd. Available at: https://carersqld.com.au/wp-content/uploads/Quality-of-Life-Audit-Report-2017.pdf (accessed on 14 December 2017). 
Whitty, J. A., Kendall, E., Sav, A., et al. (2015) Preferences for the delivery of community pharmacy services to help manage chronic conditions. Research in Social and Administrative Pharmacy 11 (2), 197-215.

Willemse, E., Anthierens, S., Farfan-Portet, M. I., et al. (2016) Do informal caregivers for elderly in the community use support measures? A qualitative study in five European countries. BMC Health Services Research 16 (1), 270.

Williams, A., Sethi, B., Duggleby, W., et al. (2016) A Canadian qualitative study exploring the diversity of the experience of family caregivers of older adults with multiple chronic conditions using a social location perspective. International Journal for Equity in Health 15 (1), 40.

Wilson, P. M., Kataria, N. \& McNeilly, E. (2013) Patient and carer experience of obtaining regular prescribed medication for chronic disease in the English National Health Service: a qualitative study. BMC Health Services Research. 13 (1), 192.

Wingham, J., Frost, J., Britten, N., et al. (2015) Needs of caregivers in heart failure management: A qualitative study. Chronic Illness 11 (4), 304-319.

World Health Organisation (2016) The growing need for long-term care: assumptions and realities. Available at:

https://www.un.org/development/desa/ageing/news/2016/09/briefing-paper-growingneed-for-long-term-care-assumptions-and-realities/ (accessed on 14 December 2017). 